T. Matsuzawa

Nagoya Math. J.

Vol. 59 (1975), 87-96

\title{
ON A HYPOELLIPTIC BOUNDARY VALUE PROBLEM
}

\author{
TADATO MATSUZAWA
}

\section{\$1. Introduction.}

This paper is devoted to the investigation of the hypoellipticity of the following first boundary value problem:

$$
\begin{aligned}
& L u=u_{t t}+\left(a(x, t) u_{x}\right)_{x}+g(x, t) u_{x t}+b(x, t) u_{x}+b^{0}(x, t) u_{t}+c(x, t) u \\
& =f(x, t) \quad \text { in } \Omega, \\
& \left.\quad u(x, t)\right|_{t=0}=0, \quad|x|<R,
\end{aligned}
$$

where $\Omega$ is an open rectangular domain in $(x, t)$-plane:

$$
\Omega=(-R<x<R) \times(0<t<T) \quad R>0, T>0 .
$$

We assume that the coefficients $a(x, t), b(x, t), b^{0}(x, t)$ and $c(x, t)$ are all $C^{\infty}$ functions in $\bar{\Omega}$ satisfying the following conditions:

$$
\operatorname{Re} a(x, t) \geqq 0 \quad \text { in } \bar{\Omega},
$$

(1.4) for all $x$ with $|x|<R$, the function $t \mapsto \operatorname{Re} a(x, t)$ has only finite zeros of order less than or equal to $\ell(\geqq 0)$ in the interval $[0 \leqq t \leqq T]$

$$
\begin{gathered}
|\operatorname{Im} a(x, t)| \leqq C^{1)} \operatorname{Re} a(x, t) \quad \text { in } \bar{\Omega}(C>0), \\
\left|\operatorname{Im} a_{x}(x, t)\right| \leqq C[\operatorname{Re} a(x, t)]^{1 / 2} \quad \text { in } \bar{\Omega}, \\
t|\operatorname{Im} b(x, t)|^{2} \leqq C \operatorname{Re} a(x, t) \quad \text { in } \bar{\Omega}, \\
|g(x, t)| \leqq \frac{\varepsilon_{1}}{2}[\operatorname{Re} a(x, t)]^{1 / 2} \quad \text { in } \bar{\Omega}, 0<\varepsilon_{1}<1, \\
\left|g_{t}(x, t)\right| \leqq C[\operatorname{Re} a(x, t)]^{1 / 2} \quad \text { in } \bar{\Omega} .
\end{gathered}
$$

We set $\tilde{\Omega}=(-R<x<R) \times[0 \leqq t<T)$. The main result of this paper is to prove the following theorem.

Received March 12, 1975.

1) We use the symbols $C, C^{1}, \ldots$ to express the different positive constants throughout this paper. 
THEOREM 1.1. Suppose that the operator $L$ given in (1.1) satisfies the condition (1.3) (1.9). Then any distribution $u \in \mathscr{D}^{\prime}(\widetilde{\Omega})$ satisfying (1.1) and $(1.2)^{2)}$ with $f(x, t) \in C^{\infty}(\widetilde{\Omega})$ must be a $C^{\infty}$ function in $\tilde{\Omega}$.

We remark that if we consider the partial differential operator of first order

$$
L_{1}=\frac{\partial}{\partial t}+i a(x, t) \frac{\partial}{\partial x}+c(x, t) \quad \text { in } \Omega,
$$

a sufficient condition of Nirenberg and Treves (cf. [10], [11]) for the operator $L_{1}$ to be hypoelliptic is expressed by (1.3) and (1.4). This is a necessary and sufficient condition when $a(x, t)$ is analytic in $\bar{\Omega}$. Our problem is motivated by this fact (cf. [6]) and the proof of Theorem 1.1 will be obtained in the following paragraphs by a refinement of the method used in [2] and [4]. For the equations of the second order with real coefficients we refer to [2] and [9].

EXAMPLES. The following operators satisfy the condition (1.3) (1.7) in a neighbourhood of the origin.

$$
L_{2}=\frac{\partial^{2}}{\partial t^{2}}+t a(x, t) \frac{\partial^{2}}{\partial x^{2}}+b(x, t) \frac{\partial}{\partial x}+b^{0}(x, t) \frac{\partial}{\partial t}+c(x, t),
$$

$\operatorname{Re} a(x, t)>0$ in $\bar{\Omega}, b, b^{0}$ and $c$ are arbitrary complex valued $C^{\infty}$ functions in $\bar{\Omega}$,

$$
\begin{aligned}
L_{3}= & \frac{\partial^{2}}{\partial t^{2}}+t^{3}[t-g(x)]^{2 \ell} \frac{\partial^{2}}{\partial x^{2}}+(1+i) t[t-g(x)]^{e} \frac{\partial}{\partial x} \\
& +b^{0}(x, t) \frac{\partial}{\partial t}+c(x, t), \\
& \ell \quad \text { integer, } \geqq 0 ; g(x) \text { is a real valued } C^{\infty} \text { function in } \\
& (-R \times x \times R), b^{0}, c \text { are arbitrary } C^{\infty} \text { functions in } \bar{\Omega} .
\end{aligned}
$$

\section{§ 2. Preliminaries for the proof of Theorem 1.1.}

LEMMA 2.1. ([9], Lemma 1.7.1) Let $a(x, t)$ be the function given in $\S 1$. Then there exists a positive constant $C$ such that

$$
\left|a_{x}(x, t)\right|^{2} \leqq C \operatorname{Re} a(x, t) \quad(x, t) \in \bar{\Omega} .
$$

Being suggested by [2] and [4], we now introduce the norm ||| |||

2) By the partial hypo-ellipticity of $L$ in $t$, condition (1.2) is meaningful in the sense of distributions (cf. [1], Ch. 4). 
and its dual norm $\|\left.|\cdot|\right|^{\prime}$ by

$$
\begin{aligned}
& \|u\|^{2}=\left\|u_{t}\right\|^{2}+\left\|\sqrt{\operatorname{Re} a} u_{x}\right\|^{2}+\|u\|^{2}, \\
& \|v\|^{\prime}=\sup _{w \in C_{0}^{\infty}(\tilde{\Omega})} \frac{|\langle v, w\rangle|}{\|w\|},
\end{aligned}
$$

where $\|\cdot\|$ is the usual $L^{2}$-norm on $\tilde{\Omega}$ and $\langle v, w\rangle$ is the value of $v \in \mathscr{D}^{\prime}(\tilde{\Omega})$ evaluated at $w$.

LEMMA 2.2. Let $L$ be the operator given in (1.1). We have the following estimate with some positive constant $C$

$$
\|v\|\|\leqq C\| v\|+\| L v\|\|^{\prime}, \quad v \in C_{0}^{\infty}(\widetilde{\Omega}), v(x, 0)=0 .
$$

Proof. Obviously we have

$$
|\langle L v, \bar{v}\rangle| \leqq\|L v\|^{\prime}|\|v\||, \quad v \in C_{0}^{\infty}(\tilde{\Omega}), v(x, 0)=0 .
$$

Next, integrating by parts, we have

$$
\begin{gathered}
-\operatorname{Re}\langle L v, \bar{v}\rangle= \\
\left\|v_{t}\right\|^{2}+\left\|\sqrt{\operatorname{Re} a} v_{x}\right\|^{2}+\operatorname{Re}\left\langle g_{t} v_{x}, \bar{v}\right\rangle+\operatorname{Re}\left\langle g v_{x}, \bar{v}_{t}\right\rangle \\
-\operatorname{Re}\left\langle b v_{x}, \bar{v}\right\rangle-\operatorname{Re}\left\langle b^{0} v_{t}, \bar{v}\right\rangle-\operatorname{Re}\langle c v, \bar{v}\rangle .
\end{gathered}
$$

For the term of the right hand side, we have for any positive number $\delta$

$$
\begin{aligned}
\left|\left\langle\operatorname{Im} b v_{x}, \bar{v}\right\rangle\right| & =\left|\left\langle t^{1 / 2} \operatorname{Im} b v_{x}, t^{-1 / 2} \bar{v}\right\rangle\right| \\
& \leqq \delta\left\langle t|b|^{2} v_{x}, \bar{v}_{x}\right\rangle+\frac{1}{\delta}\left\|t^{-1 / 2} v\right\|^{2}
\end{aligned}
$$

On the other hand, for any $\varepsilon>0$, we easily have

$$
\left\|t^{-1 / 2} v\right\|^{2} \leqq \varepsilon\left\|v_{t}\right\|^{2}+C(\varepsilon)\|v\|^{2}
$$

Thus, by virtue of the assumption (1.7), we are given the inequality

$$
\left|\left\langle\operatorname{Im} b v_{x}, \bar{v}\right\rangle\right| \leqq \delta\left\|\sqrt{\operatorname{Re} a} v_{x}\right\|^{2}+\frac{\varepsilon}{\delta}\left\|v_{t}\right\|^{2}+\frac{C(\varepsilon)}{\delta}\|v\|^{2} .
$$

For the remaining terms we have

$$
\begin{aligned}
\left|\left\langle g_{t} v_{x}, \bar{v}\right\rangle\right| & \leqq \delta\left\|\sqrt{\operatorname{Re} a} v_{x}\right\|^{2}+\frac{C(\delta)}{\delta}\|v\|^{2}, \\
\left|\left\langle g v_{x}, \bar{v}_{t}\right\rangle\right| & \leqq \varepsilon_{1}\left(\left\|\sqrt{\operatorname{Re} a} v_{x}\right\|^{2}+\left\|v_{t}\right\|^{2}\right), \\
\left|\left\langle b^{0} v_{t}, \bar{v}\right\rangle\right| & \leqq \delta\left\|v_{t}\right\|^{2}+\frac{C^{\prime}}{\delta}\|v\|^{2} \\
|\langle c v, \bar{v}\rangle| & \leqq C^{\prime \prime}\|v\|^{2} .
\end{aligned}
$$


Taking $\delta$ and $\varepsilon$ sufficiently small, we have

$$
\begin{aligned}
-\operatorname{Re}\langle L v, \bar{v}\rangle & \geqq C_{1}\|v\|\left\|^{2}-C_{2}\right\| v \|^{2} \\
& \geqq C_{1}\|v\|\left\|^{2}-C_{2}\right\| v\|\cdot\| v\|\| .
\end{aligned}
$$

This, combining with (2.3), gives the estimate (2.2).

Q.E.D.

LEMMA 2.3. Let $a(x, t)$ be as above, then we have

$$
\begin{gathered}
\left\|a_{x} v_{x}\right\|^{\prime} \leqq C\|v\|, \\
\left\|a v_{x}\right\|\left\|^{\prime} \leqq C\right\| v \|, \\
\left\|\operatorname{Im} b v_{x}\right\| \|^{\prime} \leqq C\left(\left\|v_{t}\right\|+\|v\|\right)
\end{gathered}
$$

for all $v \in C_{0}^{\infty}(\widetilde{\Omega}),(v(x, 0)=0$ for $(2.6))$ with some positive constant $C$.

Proof. For any $w \in C_{0}^{\infty}(\tilde{\Omega})$, we have

$$
\begin{aligned}
\left\langle a_{x} v_{x}, w\right\rangle & =\left\langle v_{x}, a_{x} w\right\rangle \\
& =-\left\langle v, a_{x} w_{x}\right\rangle-\left\langle v, a_{x x} w\right\rangle .
\end{aligned}
$$

Taking account of the assumption (1.6) and Lemma 2.1, we have

$$
\left|\left\langle a_{x} v_{x}, w\right\rangle\right| \leqq C\|v\| \cdot\|w\| \mid
$$

from which follows the estimate (2.4). By the same way we have (2.5). As in the proof of Lemma 2.2, we have

$$
\begin{aligned}
\left\langle\operatorname{Im} b v_{x}, w\right\rangle & =-\left\langle v, \operatorname{Im} b w_{x}\right\rangle-\left\langle v, \operatorname{Im} b_{x} w\right\rangle, \\
\left\langle\operatorname{Im} b v_{x}, w\right\rangle \mid & \leqq\left|\left\langle t^{-1 / 2} v, t^{1 / 2} \operatorname{Im} b w_{x}\right\rangle\right|+C\|v\|\|w\| \\
& \leqq C^{\prime}\left(\left\|v_{t}\right\|+\|v\|\right) \mid\|w\| \|,
\end{aligned}
$$

which imply (2.6).

Now we introduce the norm $\|\cdot\|_{(s, k)}$, with $s$ any real number and $k$ non negative integer (cf. [1], §2.6), defined by

$$
\begin{gathered}
\|v\|_{(s, k)}^{2}=(2 \pi)^{-1} \int_{0}^{\infty} \int_{R_{\xi}}|\hat{v}(\xi, t)|^{2}\left(1+|\xi|^{2}\right)^{s} d \xi d t+\sum_{j=0}^{k}\left\|D_{t}^{j} v\right\|_{L^{2}\left(R_{+}^{2}\right)}^{2}, \\
\hat{v}(\xi, t)=\int e^{-i x \xi} v(x, t) d x, \quad v \in C_{0}^{\infty}\left(\bar{R}_{+}^{2}\right) .
\end{gathered}
$$

We denote by $H_{(s, k)}\left(\bar{R}_{+}^{2}\right)$ the completion of $C_{0}^{\infty}\left(\bar{R}_{+}^{2}\right)$ in the norm $\|\cdot\|_{(s, k)}$.

LEMMA 2.4. There exists a positive constant $C$ such that

$$
\|v\|_{(1 /(\ell+1), 1)} \leqq C\|v\| \|, \quad v \in C_{0}^{\infty}(\tilde{\Omega}), v(x, 0)=0 .
$$


Proof. Since we have

$$
\begin{aligned}
\operatorname{Re} a(x, t) & \leqq C_{1} \sqrt{\operatorname{Re} a(x, t)}, \quad(x, t) \in \bar{\Omega}, \\
& \leqq C_{1}^{2}\|\| v \|^{2}, \quad v \in C_{0}^{\infty}(\tilde{\Omega}) .
\end{aligned}
$$

If we consider the differential operator

$$
L_{4}=\frac{\partial}{\partial t}+i \operatorname{Re} a(x, t) \frac{\partial}{\partial x} \quad \text { in } \tilde{\Omega},
$$

we have

$$
\left\|L_{4} v\right\|^{2}=\left\|v_{t}\right\|^{2}+\left\|\operatorname{Re} a v_{x}\right\|^{2} .
$$

On the other hand, as a particular case of Theorem I in [10], it follows that

$$
\|v\|_{(1 /(\ell+1), 1)} \leqq C_{2}\left\|L_{4} v\right\| \quad v \in C_{0}^{\infty}(\tilde{\Omega}), v(x, 0)=0
$$

with another constant $C_{2}>0$. Combining the above investigations, we have (2.7).

Q.E.D.

By using Lemma 2.2 and Lemma 2.4 we now come to the main estimate:

LEMMA 2.5. There exists a positive constant $C$ such that

$$
\|v\|_{(\varepsilon, 1)} \leqq C\left(\|v\|+\|L v\|^{\prime}\right), \quad v \in C_{0}^{\infty}(\tilde{\Omega}), v(x, 0)=0,\left(\varepsilon=\frac{1}{\ell+1}\right)
$$

LEMMA 2.6. Every $v \in H_{(0,2)}\left(R_{+}^{2}\right) \cap \mathscr{E}^{\prime}(\tilde{\Omega})$ such that $v(x, 0)=0$ and $\|L v\| \|^{\prime}<\infty$ belongs to $H_{(\varepsilon, 2)}\left(R_{+}^{2}\right)$ with $\varepsilon=\frac{1}{\ell+1}$.

Proof. The inequality (2.8) is valid for all $v \in H_{(2.2)}\left(R_{+}^{2}\right) \cap \mathscr{E}^{\prime}(\tilde{\Omega})$, $v(x, 0)=0$. Indeed, we can find a sequence $v_{j} \in C_{0}^{\infty}(\widetilde{\Omega})$ such that $v_{j}(x, 0)$ $=0, D_{x}^{\alpha} D_{t}^{\beta} v_{j}-D_{x}^{\alpha} D_{t}^{\beta} v \rightarrow 0, j \rightarrow \infty$, when $\alpha+\beta \leqq 2$. Hence $\left\|L v_{j}-L v\right\|$ $\rightarrow 0$, which implies that $\left\|L v_{j}-L v\right\|^{\prime} \rightarrow 0$. In particular,

$$
\varlimsup
$$

So it follows from (2.8) applied to $v_{j}$ that

$$
\varlimsup \overline{\lim }\left\|v_{j}\right\|_{(s, 1)} \leqq C\left(\|v\|+\|L v\| \|^{\prime}\right) \text {. }
$$

Next if $v$ satisfies the required conditions, we choose $\chi \in D_{0}^{\infty}(\tilde{\Omega})$ so that $0 \leqq \chi \leqq 1$ and $\chi=1$ in a neighbourhood $\omega$ of supp. $v$ and we set 


$$
v_{\delta}=\chi\left(1-\delta^{2} \Delta\right)^{-1} v .
$$

Here $\left(1-\delta^{2} \Delta\right)^{-1} v$ is defined as the inverse Fourier transform of $\left(1+\delta^{2}|\xi|^{2}\right)^{-1} \hat{v}(\xi, t)$ :

$$
v_{\delta}=(2 \pi)^{-1} \int_{R_{\xi}} e^{i x \xi}\left(1+\delta^{2} \xi^{2}\right)^{-1} \hat{v}(\xi, t) d \xi
$$

It is clear that $v_{\delta}$ is then in $H_{(2,2)}\left(R_{+}^{2}\right) \cap \mathscr{E}^{\prime}(\widetilde{\Omega})$, and that $v_{\delta} \rightarrow v$ in $L^{2}$ norm as $\delta \rightarrow 0$. Hence we may apply (2.8) to $v_{\delta}$ to conclude that $\|v\|_{(e, 1)}$ $<\infty$ provided that we can show that $\left.\left\|L v_{\delta}\right\|\right|^{\prime}$ remains bounded when $\delta \rightarrow 0$. To prove the last assertion we must prepare some remarks which correspond to $1^{\circ} \sim 4^{\circ}$ of [2].

$1^{\circ}$. We have

$$
\frac{1}{2} e^{-|x|}=\mathscr{F}^{-1}\left[\left(1+\xi^{2}\right)^{-1}\right]=(2 \pi)^{-1} \int_{-\infty}^{\infty} e^{i x \xi} \frac{d \xi}{1+\xi^{2}}, \quad-\infty<x<\infty .
$$

Since

$$
v_{\delta}=\left(1-\delta^{2} \Delta\right)^{-1} v(x, t)=\delta^{-1} \int K\left(\frac{x-y}{\delta}\right) v(y, t) d y, \quad K(x)=\frac{1}{2} e^{-|x|},
$$

it follows that any derivative of $\left(1-\delta^{2} \Delta\right)^{-1} v(x, t)$ decreases faster than any power of $\delta$ as $\delta \rightarrow 0$ if $(x, t) \oplus \omega$.

$2^{\circ}$. If $Q$ is a differential operator of order $j \leqq 2\left(\right.$ in $\left.\frac{\partial}{\partial x}\right)$ with coefficients in $C^{\infty}(\bar{\Omega})$, it follows that

$$
\left\|\left(1-\delta^{2} \Delta\right)^{-1} Q u\right\| \leqq C\|u\|, \quad u \in L^{2}(\tilde{\Omega}) \cap \varepsilon^{\prime}(\tilde{\Omega}) .
$$

$3^{\circ}$. When $\chi \in C_{0}^{\infty}(\tilde{\Omega})$ we have

$$
\left\|\left|\chi\left(1-\delta^{2} \Delta\right)^{-1} w\right|\right\| \leqq C\left\||w \||, \quad w \in C_{0}^{\infty}(\widetilde{\Omega}) .\right.
$$

Indeed, we have

$$
\left\|\left[\chi\left(1-\delta^{2} \Delta\right)^{-1} w\right]_{t}\right\| \leqq C\left(\left\|w_{t}\right\|+\|w\|\right), \quad w \in C_{0}^{\infty}(\tilde{\Omega})
$$

and

$$
\begin{gathered}
\left\langle\operatorname{Re} a(x, t)\left(\chi\left(1-\delta^{2} \Delta\right)^{-1} w\right)_{x}, \overline{\left.\left(\chi\left(1-\delta^{2} \Delta\right)^{-1} w\right)_{x}\right\rangle \mid}\right. \\
\leqq C\left(\|w\|^{2}+\left\|\sqrt{\operatorname{Re} a}\left(1-\delta^{2} \Delta\right)^{-1} w_{x}\right\|^{2}\right) .
\end{gathered}
$$

For the second term of the right hand side, we have 


$$
\begin{aligned}
& \left\|\sqrt{\operatorname{Re} a}\left(1-\delta^{2} \Delta\right)^{-1} w_{x}\right\|^{2} \\
& \quad=\left\|\left(1-\delta^{2} \Delta\right)^{-1} \sqrt{\operatorname{Re} a} w_{x}+\left[\sqrt{\operatorname{Re} a} D_{x},\left(1-\delta^{2} \Delta\right)^{-1}\right] w\right\|^{2} \\
& \quad \leqq 2\left\|\sqrt{\operatorname{Re} a} w_{x}\right\|^{2}+2\left\|\left[\sqrt{\operatorname{Re} a} D_{x},\left(1-\delta^{2} \Delta\right)^{-1}\right] w\right\|^{2} .
\end{aligned}
$$

By virtue of $1^{\circ}$, partial integration proves

$$
\begin{aligned}
& {\left[\sqrt{\operatorname{Re} a} D_{x},\left(1-\delta^{2} \Delta\right)^{-1}\right] w(x, t)} \\
& =\frac{A}{\delta} \int \exp \left(-\frac{|x-y|}{\delta}\right)(\sqrt{\operatorname{Re} a(x, t)}-\sqrt{\operatorname{Re} a(y, t)}) w_{y} d y \\
& =\frac{A}{\delta} \int \exp \left(-\frac{|x-y|}{\delta}\right)(\sqrt{\operatorname{Re} a(y, t)})_{y} w(y, t) d y \\
& \quad+\frac{A}{\delta^{2}} \int \exp \left(-\frac{|x-y|}{\delta}\right)(\sqrt{\operatorname{Re} a(x, t)}-\sqrt{\operatorname{Re} a(y, t)}) w(y, t) d y .
\end{aligned}
$$

By Lemma 2.1, we can see that $\sqrt{\operatorname{Re} a(x, t)}$ is uniformly Lipschitz continuous in $x$ and thus the $L^{2}$ norm of the last two terms is bounded above by $\|w\|^{2}$. This estimate combined with (2.11) gives (2.10).

Completion of the proof of Lemma 2.6. We recall that with the notations introduced above it remains to prove that $\left.\left\|L v_{\delta}\right\|\right|^{\prime}$ is bounded as $\delta \rightarrow 0$. In the neighbourhood $\omega$ of supp. $v$ we have $\left(1-\delta^{2} \Delta\right) v_{\delta}=v$ and

$$
\begin{aligned}
\left(1-\delta^{2} \Delta\right) L v_{\delta}= & \left(1-\delta^{2} \Delta\right)\left(D_{t}^{2} v_{\delta}+\left(a(x, t) v_{\delta x}\right)_{x}\right. \\
& +\left(g(x, t) v_{\delta x t}\right)+b v_{\delta x t}+b^{0} v_{\delta t}+c v_{\delta} \\
= & v_{t t}+\left(a(x, t) v_{x}\right)_{x}+g v_{x t}+b v_{x}+b^{0} v_{t}+c v \\
& -2 \delta^{2}\left(a_{x}(x, t) v_{\delta x x}\right)_{x}-\delta^{2}\left(a_{x x}(x, t) v_{\delta x}\right)_{x} \\
& -2 \delta^{2} g_{x} v_{\delta x x}-\delta^{2} g_{x x} v_{\delta x t} \\
& -2 \delta^{2} b_{x} v_{\delta x x}-\delta^{2} b_{x x} v_{\delta} x-2 \delta^{2} b_{x}^{0} v_{\delta t x} \\
& -\delta^{2} b_{x x}^{0} v_{\partial t}-2 \delta^{2} c_{x} v_{\delta x}-2 \delta^{2} c_{x x} v_{\delta} .
\end{aligned}
$$

In view of $1^{\circ}$ it follows that we have

$$
\left(1-\delta^{2} \Delta\right) L v=L v+2 \delta^{2}\left(a_{x}(x, t) v_{\delta x x}\right)_{x}+\delta^{2} B_{1} v_{\delta}+\delta^{2} B_{2} v_{\delta t}+h_{\delta},
$$

where $B_{1}$ and $B_{2}$ are second order (in $\frac{\partial}{\partial x}$ ) operators, and where $h_{\delta}$ is a function such that it vanishes in $\omega$, supp. $h_{\delta} \subset$ supp. $\chi$ and $\left\|h_{\delta}\right\| \rightarrow 0$ as $\delta \rightarrow 0$. Hence 


$$
\begin{aligned}
L v_{\delta}=\chi_{1} & \left\{\left(1-\delta^{2} \Delta\right)^{-1} L v+2 \delta^{2}\left(1-\delta^{2} \Delta\right)^{-1}\left(a_{x} v_{\delta x x}\right)_{x}\right. \\
& +\delta^{2}\left(1-\delta^{2} \Delta\right)^{-1} B_{1} v_{\delta}+\delta^{2}\left(1-\delta^{2} \Delta\right)^{-1} B^{2} v_{\delta t}+\left(1-\delta^{2} \Delta\right)^{-1} h_{\delta},
\end{aligned}
$$

where $\chi_{1}$ is a function in $C_{0}^{\infty}(\tilde{\Omega})$ which is equal to 1 in supp. $\chi$. We remark that from $3^{\circ}$ we have

$$
\left.\left\|\chi_{1}\left(1-\delta^{2} \Delta\right)^{-1} f\right\|\right|^{\prime} \leqq C\|f\| \|^{\prime}, \quad f \in \mathscr{D}^{\prime}(\tilde{\Omega}) \cap \mathscr{E}^{\prime}(\tilde{\Omega}) .
$$

Therefore, it follows that

$$
\|\| \chi_{1}\left(1-\delta^{2} \Delta\right)^{-1} L v\left|\left\|^{\prime} \leqq C\right\| L v \|\right|^{\prime} .
$$

The last three terms of (2.12) are bounded in $L^{2}$ norm in view of $2^{\circ}$ and by the assumption $v \in_{(0,2)}(\tilde{\Omega}) \cap \mathscr{E}^{\prime}(\tilde{\Omega})$. For the second term, by (2.13), $2^{\circ}$ and (2.4), we have

$$
\begin{aligned}
& \left.\left\|\chi_{1} \delta^{2}\left(1-\delta^{2} \Delta\right)^{-1}\left(a_{x} v_{\delta x x}\right)_{x}\right\|\right|^{\prime} \\
& \quad \leqq\left. C\left\|\delta^{2}\left(a_{x} v_{\delta x x}\right)_{x}\right\|\right|^{\prime} \\
& \quad \leqq 2 C\left(\left\|\delta^{2} a_{x x} v_{\delta x x}\right\|\left\|^{\prime}+\right\| \delta^{2} a_{x} D_{x} v_{\delta x x} \|\left.\right|^{\prime}\right) \\
& \quad \leqq C^{\prime}\left\|v_{\delta}\right\| \leqq C^{\prime \prime}\|v\| .
\end{aligned}
$$

This completes the proof of Lemma 2.6.

\section{§3. Proof of Theorem 1.1.}

Given a function $\psi(x, t) \in C_{0}^{\infty}(\tilde{\Omega})$ and an integer $k \geqq 2$, we may assume, by the partial hypoellipticity of $L$ in $t$ (cf. [1], $\S 4.3$ ), that $\psi u$ $\in H_{(s, k)}\left(\bar{R}_{+}^{2}\right) \cap \mathscr{E}^{\prime}(\tilde{\Omega})$ for some real number $s$. For the proof of Theorem 1.1 it suffices to show that $s$ can be replaced by $s+\varepsilon, \varepsilon=\frac{1}{\ell+1}$. Indeed, it follows that $u \in H_{(s, k)}^{\mathrm{loc}}(\widetilde{\Omega})$ for any $s$ and $k$, which means that $u \in C^{\infty}(\widetilde{\Omega})$.

Let $E$ be a pseudo-differential operator (in $x$ ) with symbol $e(\xi)=$ $\left(1+\xi^{2}\right)^{s / 2}$ (cf. [3]), and set $v=\chi E \psi u$ where $\chi \in C_{0}^{\infty}(\widetilde{\Omega})$. If we can show that $v \in H_{(\varepsilon, k)}$ for every $\chi$ and $\psi$ we will have $E \psi u \in H_{(s, 2)}^{\mathrm{loc}}(\tilde{\Omega})$, hence $u \in H_{(s+\varepsilon, 2)}^{\mathrm{loc}}$ since $E$ is elliptic. It is clear that $v \in H_{(0,2)}\left(\bar{R}_{+}^{2}\right) \cap \mathscr{E}^{\prime}(\tilde{\Omega})$, $v(x, 0)=0$, so in view of Lemma 2.6 it remains only to show that $\|L v\| \|^{\prime}<\infty$. We note that $E^{\prime}=\chi E \psi$ is a compactly supported pseudodifferential operator (in $x$ ) of order $s$ with parameter $t \geqq 0$, (cf. [3]) and $L v=L E^{\prime} u$. Taking account of $E^{\prime} L u=E^{\prime} f \in L^{2}(\widetilde{\Omega})$ and $\left\|E^{\prime} f\right\|<\infty$, it now sufficies to show that

$$
\left\|L E\left|u-E^{\prime} f\right|\right\|<\infty .
$$


We have

$$
\begin{aligned}
L E^{\prime} u-E^{\prime} f= & 2 E_{t}^{\prime} u_{t}+E_{t t}^{\prime} u+\left[a D_{x}^{2}, E^{\prime}\right] u+\left[g D_{x} D_{t}, E^{\prime}\right] u \\
& +\left[b D_{x}, E^{\prime}\right] u+\left[b^{0} D_{t}, E^{\prime}\right] u+\left[c, E^{\prime}\right] u \\
= & {\left[a D_{x}^{2}, E^{\prime}\right] u+E^{\prime \prime} u+E^{\prime \prime \prime} u_{t} }
\end{aligned}
$$

where $E^{\prime \prime}$ and $E^{\prime \prime \prime}$ are compactly supported pseudo-differential operators (in $x$ ) of order $\leqq s$ with parameter $t \geqq 0$. Obviously $\left\|E^{\prime \prime} u\right\|<\infty$ and $\left\|E^{\prime \prime \prime} u_{t}\right\|<\infty$ and $\left\|g D_{x} E_{t}^{\prime} u\right\| \|^{\prime}<\infty$ by (1.8) and (2.5), so we shall analyse the first term in the right hand side. We have

$$
\left[a D_{x}^{2}, E^{\prime}\right]=\left[a D_{x}, E^{\prime}\right] D_{x}+a D_{x} E_{x}^{\prime},
$$

and

$$
\left\|\left[a D_{x}^{2}, E^{\prime}\right] u\right\|\left\|^{\prime} \leqq\right\|\left[\left[a D_{x}, E^{\prime}\right] u_{x}\|\|^{\prime}+\left\|\left|a D_{x} E_{x}^{\prime} u \|\right|^{\prime} .\right.\right.
$$

By (2.5) the last term is estimated by $\left\|E_{x}^{\prime} u\right\|<\infty$. For any $w \in C_{0}^{\infty}(\tilde{\Omega})$ we have

$$
\begin{aligned}
& \quad\left\langle\left[a D_{x}, E^{\prime}\right] u_{x}, w\right\rangle \mid \\
& \quad \leqq\left|\left\langle\left[a D_{x}, E^{\prime}\right]_{x} u, w\right\rangle\right|+\left|\left\langle\left[a D_{x}, E^{\prime}\right] u, w_{x}\right\rangle\right| .
\end{aligned}
$$

Since the order of $\left[a D_{x}, E^{\prime}\right]_{x}$ is $\leqq s$ the first term is estimated by $C\|w\|$ with another constant $C$. Let $\sigma\left[a D_{x}, E^{\prime}\right]$ be a symbol of $\left[a D_{x}, E^{\prime}\right] . \quad A$ simple calculation (cf. [3]) proves the equality

$$
\sigma\left[a D_{x}, E^{\prime}\right]=a \cdot \sigma\left(E_{1}\right)+a_{x} \sigma\left(E_{2}\right)+E_{3},
$$

where $E_{1}, E_{2}$ and $E_{3}$ are compactly supported pseudo-differential operators (in $x$ ) of order $\leqq s$ and $\leqq s-1$, respectively. This equality leads us, by partial integration and by use of (2.4), to the following estimate

$$
\left|<\left[a D_{x}, E^{\prime}\right] u, w_{x}\right| \leqq C\||w|\| .
$$

The above investigation implies that

$$
\left\|\left[a D_{x}^{2}, E^{\prime}\right] u\right\| \|^{\prime}<\infty .
$$

Thus we have $\left\||L v \||^{\prime}<\infty\right.$ and this completes the proof of Theorem 1.1.

\section{REFERENCES}

[1] Hörmander, L.: Linear partial differential operators, Springer Verlag, 1964.

[2] Hörmander, L.: Hypoelliptic second order differential equations, Acta Math., 119 (1968), 147-171. 
[ 3 ] Hörmander, L.: Fourier integral operators, I, Acta Math., 127 (1971), 79-183.

[ 4 ] Kato, Y.: On a class of hypoelliptic differential operators, Proc. Japan Acad. 46, No. 1 (1970), 33-37.

[ 5 ] Matsuzawa, T.: Sur les équations $u_{t t}+t^{\alpha} u_{x x}=f(\alpha \geqq 0)$, Nagoya Math. J., Vol. 42 (1971), 43-55.

[6] Matsuzawa, T.: On some degenerate parabolic equations I, Nagoya Math. J. 51 (1973), 57-77, II, Nagoya Math. J. 52 (1973), 61-84.

[ 7 ] Matsuzawa, T. (with Y. Hashimoto) : On a class of degenerate elliptic equations, Nagoya Math. J. 55 (1974), 181-204.

[ 8 ] Mizohata, S.: Solutions nulles et solutions non analytiques, J. Math. Kyoto Univ. (1962), 271-302.

[9] Oleinik, O. A. and Radkevič, E. V.: Second order equations with nonnegative characteristic form, Amer. Math. Soc., 1973.

[10] Treves, F.: A new method of proof of the subelliptic estimates, Comm. Pure Applied Math., 24 (1971), 71-115.

[11] Treves, F.: Analytic-hypoelliptic partial differential equations of principal type, Comm. Pure Applied Math., 24 (1971), 537-570.

Department of Mathematics

Nagoya University

Added in proof. An investigation for the many variable cases will be given in a future publication. 